\title{
Searches for supersymmetric partners of third-generation quarks with CMS
}

\author{
Markéta Jansová* on behalf of the CMS collaboration \\ Université de Strasbourg, CNRS, IPHC UMR 7178, F-67000 Strasbourg, France \\ E-mail: marketa.jansova@cern.ch
}

\begin{abstract}
Searches for the direct third generation squark production in proton-proton collisions at $\sqrt{s}=13 \mathrm{TeV}$ are presented using data recorded by the CMS experiment corresponding to integrated luminosity of $35.9 \mathrm{fb}^{-1}$. No significant deviation from the standard model has been observed in either of the searches. The exclusion limits have been set in terms of Simplified Model Spectra on pair production of third generation squarks. The combination of analyses with final states containing 0,1 or 2 leptons excludes a stop $(\tilde{\mathrm{t}})$ mass of $1050 \mathrm{GeV}$ when the neutralino $\left(\tilde{\chi}_{1}^{0}\right)$ is massless and a neutralino mass of $500 \mathrm{GeV}$ for a stop mass of $900 \mathrm{GeV}$. The exclusion limit on the sbottom ( $\tilde{\mathrm{b}})$ goes up to a mass of $1225 \mathrm{GeV}$ when the neutralino is massless and a neutralino mass of $600 \mathrm{GeV}$ is excluded for a sbottom mass of $625 \mathrm{GeV}$.
\end{abstract}

XXV International Workshop on Deep-Inelastic Scattering and Related Subjects 3-7 April 2017

University of Birmingham, $U K$

${ }^{*}$ Speaker. 


\section{Introduction}

Supersymmetry (SUSY) is able to address the shortcomings of the Standard Model (SM) and thus it is popular beyond the Standard Model (BSM) theory. A symmetry between bosons and fermions is established by adding additional supersymmetric partners (sparticles) to the SM particles. The SM "naturalness problem", where an extreme fine tuning is needed to protect the Higgs mass from loop corrections, can be solved by canceling these contributions thanks to sparticle loops. As the loop corrections from the $3^{\text {rd }}$ generation quarks are the largest ones, the $3^{\text {rd }}$ generation squarks, stop $(\mathfrak{t})$ and sbottom $(\tilde{b})$, play an important role and are expected to have a mass within the reach of the LHC collider. This proceedings is considering only R-parity conserving scenarios, in which the sparticles are produced in pairs and the lightest supersymmetric particle (LSP) is stable and thus provides a dark matter candidate. Only models in terms of Simplified Model Spectra (SMS) are further considered. In these models the $\tilde{\mathrm{t}}$ and $\tilde{\mathrm{b}}$ are decaying to neutralino $\left(\tilde{\chi}_{1}^{0}\right)$ being LSP, possibly via an intermediate chargino $\left(\tilde{\chi}_{1}^{ \pm}\right)$. In this note the results of the searches for the $3^{\text {rd }}$ generation squarks with the CMS [1] collaboration are presented, using the full 2016 data set of proton-proton collisions at $\sqrt{s}=13 \mathrm{TeV}$ corresponding to integrated luminosity of $35.9 \mathrm{fb}^{-1}$.

\section{Stop searches}

\subsection{Stop decay channels and experimental signature}

Depending on the mass difference between the $\tilde{\mathrm{t}}$ and $\tilde{\chi}_{1}^{0}\left(\Delta m=m(\tilde{\mathrm{t}})-m\left(\tilde{\chi}_{1}^{0}\right)\right)$, several decay channels of the $\tilde{\mathrm{t}}$ are foreseen. If the $\Delta m>m(\mathrm{t})$, the $\tilde{\mathrm{t}}$ can decay to a top quark and a $\tilde{\chi}_{1}^{0}$ as shown in Fig. 1a. If $m(\mathrm{~W})+m(\mathrm{~b})<\Delta m<m(\mathrm{t})$, the decays $\tilde{\mathrm{t}} \rightarrow \mathrm{bW} \tilde{\chi}_{1}^{0}$ appear. Further lowering the mass difference of the $\tilde{\mathrm{t}}$ and $\tilde{\chi}_{1}^{0}$ below $m(\mathrm{~b})+m(\mathrm{~W})$ results in the four body decays or in final states with a c quark and a $\tilde{\chi}_{1}^{0}$, presented in Fig. $1 \mathrm{~b}$. If the $\tilde{\chi}_{1}^{ \pm}$is also considered in the decay cascade, the decay shown in Fig. 1c of a $\tilde{\mathrm{t}}$ to a b quark and a $\tilde{\chi}_{1}^{ \pm}$, where the $\tilde{\chi}_{1}^{ \pm}$decays to a W boson and a $\tilde{\chi}_{1}^{0}$, is considered. In this case the $\tilde{\chi}_{1}^{ \pm}$mass is fixed to $m\left(\tilde{\chi}_{1}^{ \pm}\right)=0.5\left(m(\tilde{\mathrm{t}})-m\left(\tilde{\chi}_{1}^{0}\right)\right)$.

The typical signal signature contains multiple jets $\left(N_{J}\right)$, b-jets $\left(N_{b}\right)$ and a large missing transverse energy $\left(E_{T}^{\text {miss }}\right)$ from neutralinos and eventual neutrino(s). Depending on the decay of the $\mathrm{W}$ bosons, the final state may consist in zero to two leptons. Based on these observations and other topological and kinematical variables, the preselection criteria are created to reject the most of the background.

The signal final state kinematics differ for low and high $\Delta m$. In the regime where $\Delta m$ is low, the $\tilde{\mathrm{t}}$ decay products are soft and the kinematics is similar to the background one. To produce sufficient $E_{T}^{\text {miss }}$, the stop system must be boosted against an initial state radiation (ISR). The presence of the ISR is leading to the requirement of an additional non b-tagged jet in the event. For low $\Delta m$ signals, soft b-tagging technique has been introduced by several analyses, based on the presence of a secondary vertex originating from a B-meson, not reconstructed into a jet. In the opposite extreme, when the $\Delta m$ is large, the presence of boosted topologies is expected and it is targeted by dedicated taggers. Knowing the signal and the standard model background differences, the categorization of events in several signal regions is designed to enhance the sensitivity of searches as well as to distinguish between different signal scenarios and possibly different backgrounds. 


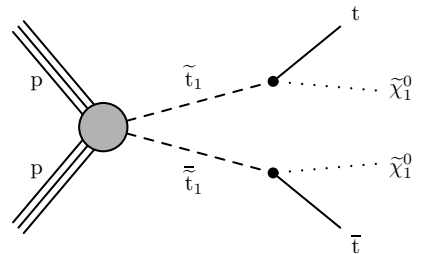

(a)

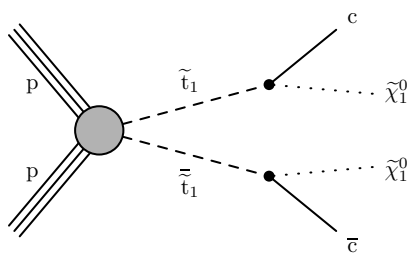

(b)

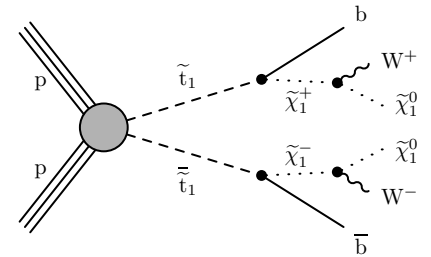

(c)

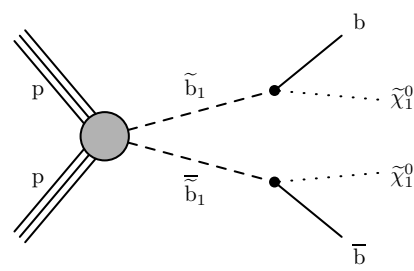

(d)

Figure 1: SUSY SMS Feynman diagrams of the $\tilde{t}$ and $\tilde{b}$ pair production for the decay modes considered in this proceedings. (1a) pp $\rightarrow \tilde{\tilde{\mathrm{t}}} \rightarrow \mathrm{t} \tilde{\chi}_{1}^{0} \overline{\mathrm{t}} \tilde{\chi}_{1}^{0},(1 \mathrm{c}) \mathrm{pp} \rightarrow \tilde{\tilde{\mathrm{t}}} \rightarrow \mathrm{b} \tilde{\chi}_{1}^{+} \overline{\mathrm{b}} \tilde{\chi}_{1}^{-}$, (1b) $\mathrm{pp} \rightarrow \tilde{\tilde{\mathrm{t}}} \rightarrow \mathrm{c} \tilde{\chi}_{1}^{0} \overline{\mathrm{c}} \tilde{\chi}_{1}^{0}$, (1d) $\mathrm{pp} \rightarrow \tilde{\mathrm{b}} \tilde{\mathrm{b}} \rightarrow \mathrm{b} \tilde{\chi}_{1}^{0} \overline{\mathrm{b}} \tilde{\chi}_{1}^{0}$.

\subsection{Background composition and estimation in stop searches}

After implementing the analysis preselection, the background composition differs with the required multiplicity of leptons $\left(N_{\ell}\right)$ in the final states. For the hadronic final state [2] the dominant contribution, referred as "lost lepton", is coming from processes (mainly $t \bar{t}, \mathrm{~W}+\mathrm{jets}$ ) in which a single generated charged lepton is lost because it is out of the kinematical acceptance or is misreconstructed. The sub-leading background consists of $\mathrm{Z}+\mathrm{jets}$ and $\mathrm{t} \overline{\mathrm{Z}} \mathrm{Z}$ processes with the $\mathrm{Z}$ boson decaying to neutrinos. The other backgrounds are QCD multijets and the rare backgrounds from dibosons. In the case of one lepton in the final state [3], the major background arises from $\bar{t} \bar{t}$ and tW production decaying to two leptons, where one of them is lost, again referred as "lost lepton". The $\mathrm{W}+$ jets process decaying to one lepton is the sub-leading background. The remaining substantial contribution to the SM backgrounds is composed by t⿱t$Z$ and $\mathrm{WZ}$ in dineutrino final state and $t \bar{t}$ decaying to one lepton. For the dilepton final state [4] the single top and $\bar{t} \bar{t}$ productions with severely mismeasured momenta or misidentified leptons are the dominant backgrounds. The residual contribution is formed by the top quark pair production with an associated Z,W or Higgs boson, Drell-Yann, diboson and multiboson processes.

\subsection{Stop search in the hadronic final state $(0 \ell)$}

The fully hadronic search [2] is targeting various signal scenarios with two separate optimization for low $\Delta m$ and moderate to high $\Delta m$. All the signal regions are orthogonal and statistically combined to obtain the exclusion limits. In the case of high $\Delta m$, expected boosted topologies are targeted by dedicated taggers. For low $\Delta m$ signals, tagging of boosted topologies is used to significantly reduce the t⿱t background. The soft b-tagging and presence of the ISR jet(s) requirement are also introduced in low $\Delta m$ signals to gain in signal sensitivity. 
In the SM processes, with the top quarks decaying hadronically, usually 3 separate jets are expected - one b-jet and two jets coming from the W boson. For the signals with the high $\Delta m$ the top quarks are considerably boosted and in consequence the jets from the $\mathrm{W}$ boson or all three jets from the top quark can be merged. The first topology is targeted by a W-tagger, the second by a top-tagger. Moderate $\Delta m$ signals are resulting in a three resolved jets topology, targeted by a resolved top-tagger.

The decay products of the boosted top quark with transverse momentum $p_{T}>400 \mathrm{GeV}$ or of the $\mathrm{W}$ boson with $p_{T}>200 \mathrm{GeV}$ are merged into one fat jet, reconstructed by an anti- $k_{t}$ clustering algorithm with a distance parameter of 0.8 . The re-clustering with Cambridge-Aachen algorithm is done for each fat jet to reveal its substructure. The fat jets are groomed using "soft drop algorithm" to remove soft and wide angle radiation. The final top quark (W boson) candidates must satisfy requirement to have a soft-drop mass $m_{S D}>110 \mathrm{GeV}\left(50<m_{S D}<110 \mathrm{GeV}\right)$. A multivariate technique (BDT) is used to create two separate identification algorithms to distinguish between merged tops (Ws) and fakes.

\subsection{Stop search in the final state with one lepton $(1 \ell)$}

The target of this analysis is the search for the stop pairs in a final state with one electron or muon [3]. In this search two sets of signal regions are defined, first the "standard" regions designed to cover the majority of the $\Delta m$ plane and secondly the "compressed" ones optimized for the $\tilde{\mathrm{t}} \rightarrow \mathrm{t} \tilde{\chi}_{1}^{0}$ signals where $\Delta m \sim m_{\mathrm{t}}$. The "compressed" analysis approach is used only for signals where both $\tilde{\mathrm{t}}$ decay into $\mathrm{t} \tilde{\chi}_{1}^{0}$ in the range $100<\Delta m<225 \mathrm{GeV}$ and it relies on the presence of ISR jet(s).

After a preselection in $N_{\ell}, N_{b}$ and $E_{T}^{\text {miss }}$, the remaining majority of $\mathrm{t} \overline{\mathrm{t}} \rightarrow 1 \ell$ and $\mathrm{W}+$ jets backgrounds is rejected by the requirement on the transverse mass $\left(M_{T}\right)$ of lepton-missing transverse momentum system. For these backgrounds, the neutrino from the leptonic decay of $\mathrm{W}$ boson provides the main source of $E_{T}^{\text {miss }}$, and thus $M_{T}$ has an endpoint at the $\mathrm{W}$ boson mass. Further suppression of the lost lepton background is achieved by the requirement on the azimuthal angle between $\vec{E}_{T}^{m i s s}$ and the closest jet from the two leading jets $\left(\Delta \phi\left(E_{T}^{\text {miss }}, J_{1,2}\right)\right)$. The definition of the "standard" signal regions is provided by categorization in $E_{T}^{\text {miss }}, N_{J}$, the invariant mass of the lepton and the closest b-tagged jet $\left(M_{\ell b}\right)$, and a modified version of the topness variable $\left(t_{m o d}\right)$ revealing how well the event agrees with the $\mathrm{t} \overline{\mathrm{t}} \rightarrow 2 \ell$ hypothesis. The advantage of $t_{\text {mod }}$, also valid for $M_{\ell b}$ variable, is its discriminating power between different signal scenarios. In general four jets are expected but a category with 2-3 jets is also added to cover topologies with merged or soft jets.

\subsection{Stop search in the dilepton final state $(2 \ell)$}

This analysis [4] presents the search for the stop pair production in the final state with oppositecharge dilepton of same or opposite flavor. The basic variable referred as $M_{T 2}(\ell \ell)$, used for both preselection and categorization, is analogical to $M_{T}$ variable. The $M_{T 2}(\ell \ell)$ variable has an endpoint at the $\mathrm{W}$ boson mass for backgrounds where the main sources of $E_{T}^{\text {miss }}$ come from the leptonic decays of $\mathrm{W}$ boson. When adding the b-jets, an endpoint at the top mass is present for backgrounds containing two $t \bar{t}$ decaying leptonically and with no other source of $E_{T}^{\text {miss }}$. 


\subsection{Interpretations on the stop production}

In the three presented stop searches no significant deviation from the standard model is observed, therefore exclusion limits on the direct stop pair production have been set in terms of SMS for different decay channels. The expected and observed limits set by the three analyses $[2,3,4]$ and their statistical combination are shown in Fig. 2. For the decay mode $\tilde{\mathfrak{t}} \rightarrow \mathrm{t} \tilde{\chi}_{1}^{0}$ (shown in Fig. 2a), the combined observed limit, mainly driven by the fully hadronic search, excludes a $\tilde{\mathrm{t}}$ mass up to $1050 \mathrm{GeV}$ for a massless $\tilde{\chi}_{1}^{0}$ and a $\tilde{\chi}_{1}^{0}$ mass of $500 \mathrm{GeV}$ for a $\tilde{\mathrm{t}}$ mass of $900 \mathrm{GeV}$. The exclusion limits for the $\tilde{\mathrm{t}} \rightarrow \mathrm{b} \tilde{\chi}_{1}^{ \pm}$model are shown in the Fig. $2 \mathrm{~b}$. In this case the strongest limit on the $\tilde{\mathrm{t}}$ and $\tilde{\chi}_{1}^{0}$ masses is put by the $1 \ell$ analysis. The combination extends the exclusion by about additional $50 \mathrm{GeV}$ for both $\tilde{\mathrm{t}}$ and $\tilde{\chi}_{1}^{0}$ masses with respect to $1 \ell$ analysis, leading to an exclusion of the $\tilde{\mathrm{t}}$ mass of $1000 \mathrm{GeV}$ for a massless $\tilde{\chi}_{1}^{0}$ and a $\tilde{\chi}_{1}^{0}$ mass of $450 \mathrm{GeV}$ for a $\tilde{\mathrm{t}}$ mass of $900 \mathrm{GeV}$.

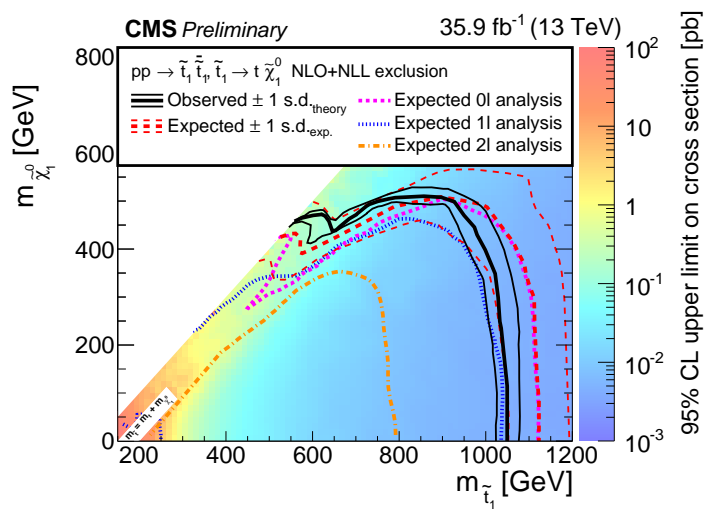

(a)

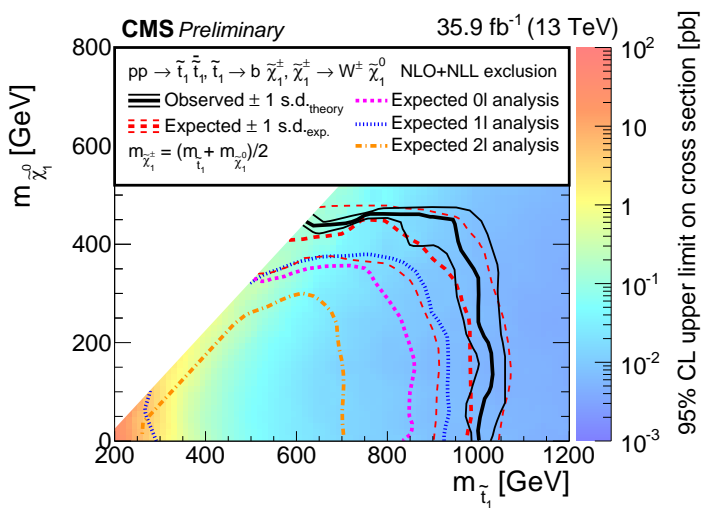

(b)

Figure 2: Exclusion limits at 95\% CL for the direct production of the stop pairs assuming $100 \%$ branching ratio for (2a) into $\tilde{\chi}_{1}^{0}$ and (2b) into $\mathrm{b} \tilde{\chi}_{1}^{ \pm}$. The $95 \% \mathrm{CL}$ upper limit on the cross section times branching ratio is indicated for all the points in the $2 \mathrm{D}$ plane $m\left(\tilde{\mathrm{t}}_{1}\right)$ vs. $m\left(\tilde{\chi}_{1}^{0}\right)$. The expected limits from the fully hadronic [2], $1 \ell$ [5] and $2 \ell$ [4] analyses are shown in magenta, blue and yellow dashed lines, respectively. The combined 95\% CL expected (in red dashed) limit [5] is shown with $\pm 1 \sigma$ experimental uncertainties while the $95 \% \mathrm{CL}$ observed limit (in black) is with $\pm 1 \sigma$ theoretical uncertainties. The area on the left from the black observed limit curve is the excluded area at $95 \%$ CL.

\section{Sbottom searches}

The production of both third generation squarks is also searched in fully hadronic final states with heavy flavor jets [6]. The dedicated search is targeting stop signal in the decay channel $\tilde{t} \rightarrow c \tilde{\chi}_{1}^{0}$ shown in Fig. $1 \mathrm{~b}$ and sbottom signal in final state $\tilde{b} \rightarrow b \tilde{\chi}_{1}^{0}$ as displayed in Fig. 1d. The search is optimized for both the high and the low $\Delta m$ signals, where in the later case specific objects taggers, c-tagger and soft b-tagger, are employed as well as the requirement on the presence of the ISR jet(s). In this search no significant deviation from the standard model has been found and exclusion limits have been set for both $\tilde{\mathrm{t}}$ and $\tilde{\mathrm{b}}$ production as shown in Fig. 3. A $\tilde{\mathrm{b}}$ mass of $1225 \mathrm{GeV}$ is excluded when the $\tilde{\chi}_{1}^{0}$ is massless as well as $\tilde{\chi}_{1}^{0}$ mass of $600 \mathrm{GeV}$ when the $\tilde{b}$ mass is $625 \mathrm{GeV}$. 


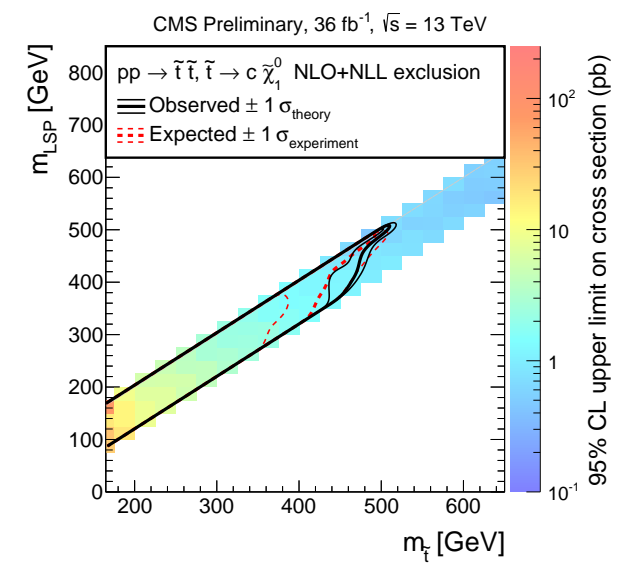

(a)

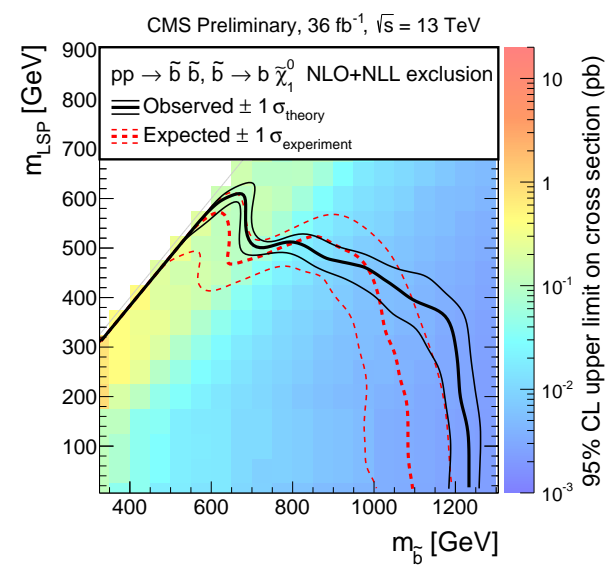

(b)

Figure 3: Exclusion limits at 95\% CL for the direct production of the stop and sbottom pairs for the decay modes (3a) $\tilde{\mathrm{t}} \rightarrow \mathrm{c} \tilde{\chi}_{1}^{0}$ and (3b) $\tilde{b} \rightarrow \mathrm{b} \tilde{\chi}_{1}^{0}$ [6]. The 95\% CL upper limit on the cross section times branching ratio is indicated for all the points in the 2D plane $m(\tilde{\mathbf{t}})$ or $m(\tilde{\mathbf{b}})$ vs. $m\left(\tilde{\chi}_{1}^{0}\right)$. The 95\% CL expected (red dashed) limit is shown with $\pm 1 \sigma$ experimental uncertainties while the $95 \%$ CL observed limit (black) is with $\pm 1 \sigma$ theoretical uncertainties.

\section{Conclusions}

The latest results of $3^{\text {rd }}$ generation SUSY searches with $35.9 \mathrm{fb}^{-1}$ of $13 \mathrm{TeV}$ CMS data have been presented. No significant deviation from the standard model has been observed by any of the analyses and the exclusion limits on $3^{\text {rd }}$ generation squarks and neutralino masses have been set. Various new techniques, such as soft b-tagging and top-tagging, have been used to increase the sensitivity of the searches far beyond the scaling with luminosity. Large effort has also been put on improving the sensitivity for the signal kinematics where $\Delta m$ is low.

\section{References}

[1] S. Chatrchyan et al. [CMS Collaboration], The CMS Experiment at the CERN LHC, JINST 3, S08004 (2008).

[2] CMS Collaboration [CMS Collaboration], Search for direct top squark pair production in the all-hadronic final state in proton-proton collisions at sqrt( $s)=13 \mathrm{TeV}$, CMS-PAS-SUS-16-049.

[3] A. M. Sirunyan et al. [CMS Collaboration], Search for top squark pair production in pp collisions at sqrt(s)=13 TeV using single lepton events, arXiv:1706.04402 [hep-ex].

[4] CMS Collaboration [CMS Collaboration], Search for direct stop pair production in the dilepton final state at $\sqrt{s}=13 \mathrm{TeV}$, CMS-PAS-SUS-17-001.

[5] CMS Collaboration [CMS Collaboration], Search for top squark pair production in the single lepton final state in pp collisions at $\sqrt{s}=13 \mathrm{TeV}$, CMS-PAS-SUS-16-051.

[6] CMS Collaboration [CMS Collaboration], Search for direct production of bottom and top squark pairs in proton-proton collisions at $\sqrt{s}=13 \mathrm{TeV}$, CMS-PAS-SUS-16-032. 